\title{
Multispacecraft observations of the hard X-ray emission from the giant solar flare on 2003 November 4
}

\author{
S. R. Kane, J. M. McTiernan, and K. Hurley
}

\author{
Space Sciences Laboratory, 7 Gauss Way, University of California, Berkeley, 94720-7450, USA \\ e-mail: sharadkane12@msn.com
}

Received 19 August 2004 / Accepted 20 December 2004

\begin{abstract}
The hard X-ray emission from the "giant" solar flare on 4 November 2003 ( 1947 UT) was observed by the hard $\mathrm{X}$-ray/gamma ray spectrometer on the Ulysses spacecraft located at $\sim 114$ degrees west of the Sun-Earth line at a distance of 5.28 AU from the Sun. A "small" hard X-ray burst during the early rise of the giant flare and a part of the decay of the hard X-ray emission associated with the giant flare were also observed by the hard X-ray imager on the RHESSI satellite located near the Earth. The maximum of the hard X-ray emission during the giant flare could not be observed by the RHESSI instrument because of satellite night. The flare view angles for Ulysses and RHESSI were $\sim 31^{\circ}$ West and $83^{\circ}$ East respectively. The $\mathrm{H}-\alpha$ flare (importance 3B) was located at S19 W83 in the active region NOAA 10486. GOES observations of the associated soft X-ray emission saturated during the period 1943-1958 UT. It has been estimated that the soft X-ray maximum occurred at $\sim 1947$ UT with peak flux equivalent to NOAA class $\geqq X 28$. OVSA radio observations show that the flare produced intense microwave emission, the peak flux at $15.4 \mathrm{GHz}$ being $\sim 60000$ sfu at $\sim 1945$ UT. Intense type II, III and IV radio bursts have also been reported at metric and decimetric wavelengths. RHESSI has provided 10-100 keV X-ray images and spectra for 1930-1936 UT (the early rise of the flare) and 10-30 keV images and spectra for 2016-2114 UT (a part of the late decay of the flare). Ulysses observed an increase in 25-150 keV X-rays from 1933 UT to 2015 UT with the maximum at 1944 UT, almost simultaneously with the maximum in $15.4 \mathrm{GHz}$ radio emission and $\sim 3$ min before the maximum in the soft X-ray emission. This indicates that the X-ray emission observed by Ulysses was mostly non-thermal. An assumed hard X-ray spectrum of $\sim E^{-3.5}$ photons $\mathrm{cm}^{-2} \mathrm{~s}^{-1} \mathrm{keV}^{-1}$ for $>20 \mathrm{keV}$ photons leads to an energy dissipation rate of $\sim 6.4 \times 10^{31} \mathrm{erg} \mathrm{s}^{-1}$ at the time of the hard X-ray maximum. The total energy in $>20 \mathrm{keV}$ electrons released during the flare is estimated to be $\sim 1.3 \times 10^{34} \mathrm{erg}$.
\end{abstract}

Key words. Sun: activity - Sun: flares - Sun: X-rays - gamma rays

\section{Introduction}

The general picture of a solar flare is one in which a large release of energy occurs in a region with a strong magnetic field, resulting in the production of energetic particles (e.g. Kahler 1992). In the vast majority of the flares, it appears that the magnetic energy in the active region greatly exceeds the energy needed to account for all the manifestations of the flare, from electromagnetic energy release to coronal mass ejections. In the previous solar cycle, however, numerous flares were observed whose energy releases appeared to be comparable to the energies of their associated active regions. Somewhat ironically, these observerations were carried out with a small detector in interplanetary space; its size and distance from the Sun made it possible to unravel the effects of saturation, which, along with Earth occultations, often confound the measurements made with instruments in Earth orbit. These large flares are clearly quite rare, and often are difficult to analyze in detail, since complementary observations in other energy ranges are frequently incomplete. It is therefore important to study as many of them as possible, in order to obtain the fullest picture of them possible. In this article we describe an event which was observed in the present solar cycle (cycle 23), which has characteristics similar to the large events of the previous cycle. The present observations appear to confirm many of the previous findings, suggesting again that some flares in each cycle release energies which are difficult to reconcile with the stored magnetic energy in their active regions.

During a period of six months near the maximum in the last solar activity cycle (cycle 22), eleven very large solar flares were observed by the hard X-ray spectrometer on the interplanetary spacecraft Ulysses (Kane et al. 1995). The gamma ray emission associated with some of these flares was also observed by some near-Earth spacecraft (e.g. Barat et al. 1994; Vilmer et al. 1999). In several of the eleven flares observed by Ulysses, the intensity of both the soft and hard X-ray emission was so large that the X-ray instruments on near-Earth spacecraft such as GOES were severely saturated and hence could not make meaningful measurements of the flare. On the other hand, the smaller $\left(20 \mathrm{~cm}^{2}\right)$ instrument on the Ulysses spacecraft, located at a distance of $\sim 3.7$ AU from the Sun, was exposed to a much smaller X-ray flux and hence was considerably less affected by saturation and pulse pileup effects. The Ulysses 
measurements indicated that, in each of the eleven solar flares, the $\geqq 20 \mathrm{keV}$ non-thermal electrons at the Sun dissipated energy in the flare region at a rate of $\geqq 10^{31} \mathrm{erg} \mathrm{s}^{-1}$, the total energy dissipated during one flare being $10^{33}-10^{34} \mathrm{erg}$. For comparison, the energy released during these flares was one to two orders of magnitude larger than the energy released during some "large" flares studied earlier, e.g. the flare on 1972 August 4 (Lin \& Hudson 1976). Therefore these eleven flares were identified as "giant" flares, which require that essentially all the available energy in the relevant active region be released during the short duration of a single flare.

Because of the implications of such a large rate of energy release for the models of particle acceleration and energy release in solar flares, it is important that the past observations of giant solar flares be repeated and confirmed during the present solar cycle (cycle 23). Such an opportunity occurred recently during the very large solar flare on 2003 November 4, which seems to have many of the characteristics of a giant flare. In addition to a very large soft X-ray burst of GOES class $>$ X28 (Kiplinger \& Garcia 2004), this flare produced intense radio emission at frequencies as high as $405 \mathrm{GHz}$ (Kaufmann et al. 2004). The Ulysses instrument measured the counting rate of 25-150 keV X-rays during the entire event. Complementary information was provided by the imaging hard X-ray spectrometer on the Ramaty High Energy Solar Spectroscopic Imager (RHESSI), which obtained images and spectra of 10-200 keV $\mathrm{X}$-rays, albeit only during the early rise and late decay phases of the flare. Here we present the hard X-ray observations of this flare and compare them with the observations of giant solar flares made in the past.

\section{Hard X-ray instruments on Ulysses and RHESSI}

The hard X-ray observations reported here were made with instruments aboard two spacecraft, Ulysses and RHESSI. The Solar X-Ray/Cosmic Gamma-Ray Burst Experiment on Ulysses, which has been in operation since its launch in 1990, has been described in general by Hurley et al. (1992), and, for the case of solar flare X-ray observations, by Kane et al. (1993). The sensor consists of two hemispherical CsI(Tl) scintillators which present an effective area of $\sim 20 \mathrm{~cm}^{2}$ in any direction. The lower energy threshold, determined by the entrance window, is $\sim 11 \mathrm{keV}$. The overall detection efficiency at that energy is $\sim 10 \%$. Normally the instrument operates in the "real time mode" where the integral counting rate in the nominal $25-150 \mathrm{keV}$ energy range is continuously recorded. The time resolution is $0.25-2.00 \mathrm{~s}$, depending on the telemetry rate. Following a rapid increase in the counting rate, as in the case of a cosmic gamma-ray burst or a solar flare, the "burst mode" is triggered, where X-ray spectra in the nominal 15$150 \mathrm{keV}$ range are saved in the memory to be read back in the telemetry at a later time. During this readout time, no additional triggers can occur, but the experiment continues to accumulate data in the real-time mode. Consequently, the spectrum of an event may not be available, even though its integral counting rate is recorded. An on-board ${ }^{241} \mathrm{Am} \mathrm{X}$-ray source provides $60 \mathrm{keV}$ X-rays for daily in-flight calibrations. The instrument has also been inter-calibrated in the past with other hard X-ray spectrometers such as the one on Yohkoh (Kane et al. 1998). A recent comparison with RHESSI measurements indicates that the inter-calibration of these two instruments is also satisfactory.

The response of the Ulysses instrument to an intense flux of thermal or non-thermal X-rays has been discussed in detail earlier (Kane et al. 1995). The estimated response is based on the calibration of the instrument in the laboratory and calculations using a Monte Carlo model. At the peak of the flare considered here, the uncorrected count rate was 69108 counts s $^{-1}$, and the dead time correction reached a factor of 6.97. (By way of comparison, the correction reached a factor of approximately 147 for the 1991 June 01 flare.) Buffer overflow caused the counters to roll over numerous times during the rise and decay phases; these rollovers are characterized by rapid, "sawtooth" increases and decreases in the apparent counting rate, and are easily tracked and corrected for. The integral rate data presented here include corrections for both effects. The times and fluxes have also been normalized to the Earth's distance from the Sun.

RHESSI has been described extensively in the literature (cf. Lin et al. 2002). The basic spectral and temporal resolution of the present observations is $\sim 2 \mathrm{keV}$ and $4 \mathrm{~s}$ respectively. The $10-200 \mathrm{keV}$ X-ray images relevant to the present study were obtained with a spatial resolution of 4-6" and were averaged over time intervals of 1-6 min. In order to reduce the incident photon flux to a level suitable for measurements by the instrument, thick and/or thin shutters (attenuators) are moved automatically (or on command) into the path of the X-rays incident on the detector. During the 2003 November 4 flare, the attenuator mode switched between A1 (thin shutter) and A3 (thick and thin shutters). There was no change in the status of the attenuator or decimation during the time intervals used for constructing the spectra and images presented in this paper.

\section{Observations}

The very large solar flare of 2003 November $4(\sim 1947$ UT) occurred in the active region NOAA 10486. The H- $\alpha$ flare (importance 3B) was located near the west limb of the Sun at S19, W83. The associated $15 \mathrm{GHz}$ radio burst, observed by the Owens Valley Solar Array (OVSA), reached a peak flux of $60000 \mathrm{sfu}$ at $\sim 1945 \mathrm{UT}$. The X-ray emission associated with the flare was observed by instruments aboard the interplanetary spacecraft Ulysses and the Earth-satellites RHESSI and GOES. The locations of these spacecraft (projected onto the ecliptic plane) relative to the Sun-Earth line and the location of the flare on the Sun are shown schematically in Fig. 1. Ulysses was located at $\sim 24^{\circ}$ behind the west limb at a distance of 5.3 AU from the Sun. The flare view angles (that is, the spacecraft-Sun center- flare region angles) for the Ulysses and RHESSI/GOES instruments were $\sim 31^{\circ}$ West and $\sim 83^{\circ}$ East respectively. The flare was therefore in full view of the Ulysses instrument. Moreover, the X-ray flux incident on the Ulysses instrument was smaller than that at the near-Earth instruments by a factor of 27.6. The flare was also in full view of both the GOES and RHESSI instruments provided that the X-ray source did not extend spatially beyond the west limb of the Sun. 
LOCATION OF ULYSSES: 4 NOV 2003 ( 1945 UT)

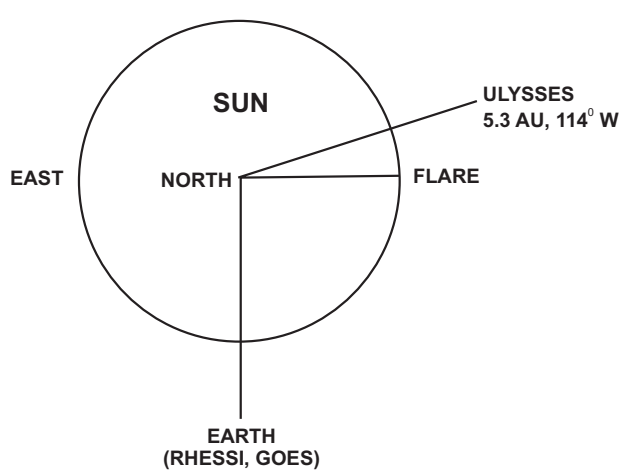

Fig. 1. The locations of the Ulysses, RHESSI and GOES spacecraft (projected onto the ecliptic plane) relative to the Sun-Earth line. The location of the 2003 November 4 flare on the solar disk is also shown.

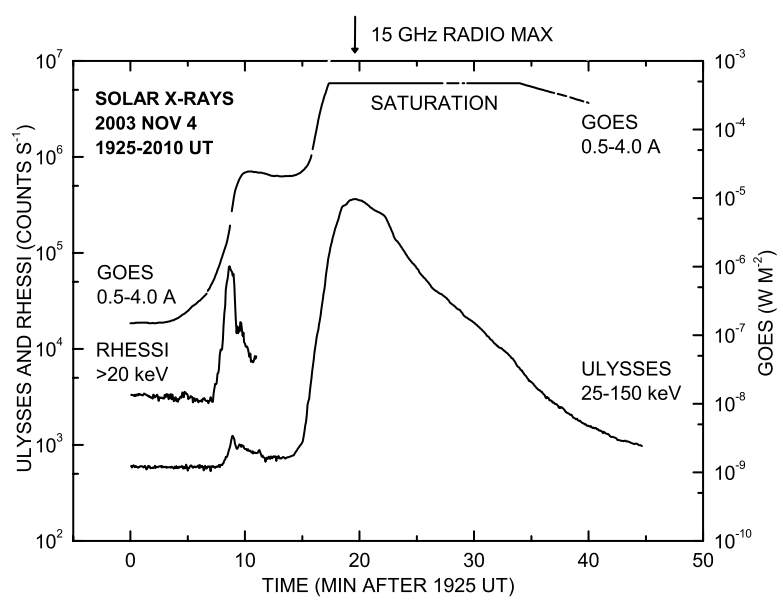

Fig. 2. From top to bottom, the time histories are shown for (1) the $0.5-4.0 \AA$ X-ray emission observed by the GOES instrument, (2) the integral counting rate of $25-150 \mathrm{keV} \mathrm{X}$-rays observed by the Ulysses instrument at the location of Ulysses (5.3 AU from the Sun), and (3) the integral counting rate of X-rays $>20 \mathrm{keV}$ observed by RHESSI. For reference, the time of maximum for the $15 \mathrm{GHz}$ radio emission observed by the Owens Valley Solar Array (OVSA) is indicated by an arrow at the top of the figure. After 1936 UT RHESSI was close to satellite night and data are not shown.

Figure 2 shows the time history of (1) the 0.5-4.0 $\mathrm{X}$-ray emission observed by the GOES instrument, (2) the 25-150 keV X-ray count rate observed by Ulysses at 5.3 AU, and (3) the integral counting rate of X-rays $>20 \mathrm{keV}$ observed by RHESSI. For reference, the time of maximum for the $15 \mathrm{GHz}$ radio emission observed by OVSA is indicated by an arrow at the top of the figure. The attenuator mode for RHESSI switched between $\mathrm{A} 1$ and $\mathrm{A} 3$ during the observation period. The rates shown are normalized to attenuator mode A3.

The soft X-ray burst associated with the flare has been classified as GOES class X28. The GOES observations (Fig. 2) show an initial small maximum at $\sim 1935$ UT followed by a rise towards the main maximum. However, the GOES instrument saturated several minutes before reaching the soft X-ray maximum and remained saturated for $\sim 15 \mathrm{~min}$. Although the exact time of the soft $\mathrm{X}$-ray maximum could not be determined, it appears that the soft X-ray emission reached its maximum several minutes after the maxima in the hard X-ray and microwave radio emissions. This is confirmed by the additional analysis of the GOES observations conducted by Kiplinger \& Garcia (2004). Although it is not possible, strictly speaking, to correct the count rates for the effects of saturation, they have compared the time profile to that of other, unsaturated events to get a picture of the overall behavior. They have estimated that the soft X-ray flare was probably of GOES class X30.6 with a maximum at 1947 UT. According to their analysis, a maximum temperature of $4.2 \times 10^{7} \mathrm{~K}$ probably occurred at $\sim 1942 \mathrm{UT}$ and the emission measure probably reached its maximum value of $\sim 1.6 \times 10^{51} \mathrm{~cm}^{-3}$ at $\sim 1948: 40 \mathrm{UT}$.

Ulysses observations of the flare consist of the $25-150 \mathrm{keV}$ integral counting rate covering the period 1920-2100 UT (Fig. 2). A significant increase above the background was detected only during the period 1932-2020 UT. No spectra were recorded during this period, as the instrument was triggering continuously on solar particles from a previous event. However, as we show below, it is nevertheless possible to obtain reasonable upper and lower limits to the X-ray flux. After a small maximum at $\sim 1933$ UT, the Ulysses counting rate increased rapidly to a maximum at $\sim 1944$ UT almost simultaneously with the $15 \mathrm{GHz}$ radio maximum. By the time the GOES instrument recovered from saturation, the Ulysses counting rate had already decreased by a factor of $\sim 100$ from its maximum value.

\section{The small hard X-ray burst}

RHESSI observed the Sun from 1925 UT to 1936 UT (Fig. 2); after 1936 UT RHESSI was close to satellite night. Also, some data were probably contaminated by terrestrial particles. Therefore RHESSI data from 1936 UT to 1956 UT could not be included in this study. RHESSI observations thus covered the early rise and late decay of the flare but did not cover the principal maximum. The Ulysses and RHESSI counting rates during the small initial hard X-ray burst are shown in Fig. 3. Although there is a general similarity between the two lightcurves, the times of the maxima and the rates of rise and decay seem to differ significantly. It appears that although most of the hard $\mathrm{X}$-ray source was in full view of both the instruments, some parts of the source were partially occulted from the view of RHESSI.

In order to compare further the RHESSI and Ulysses observations of the small X-ray burst, a double power law photon spectrum of the following form was fitted to the RHESSI observations: $J(E) \propto E^{-\gamma}$ photons $\mathrm{cm}^{-2} \mathrm{~s}^{-1} \mathrm{keV}^{-1}$, where $\gamma=\gamma_{1}$ for $E \leqq E_{\mathrm{br}}$ and $\gamma=\gamma_{2}$ for $E>E_{\mathrm{br}}$. Here $E_{\mathrm{br}}$ is the breakpoint photon energy $(\mathrm{keV})$. The expected counting rate of the Ulysses instrument due to this spectrum was then computed and compared with the observed counting rate. This procedure is similar to one used earlier by Trottet et al. (2003) in the analysis of the giant solar flare on 1991 June 30. The results obtained for the 2003 November 4 flare were as follows: (a) for the period 1932:40-1933:40 UT, which covers the onset through the first, small maximum, the spectral parameters were $\gamma_{1}=4.47$, $\gamma_{2}=4.82$, and $E_{\mathrm{br}}=49.0 \mathrm{keV}$. The computed and observed 


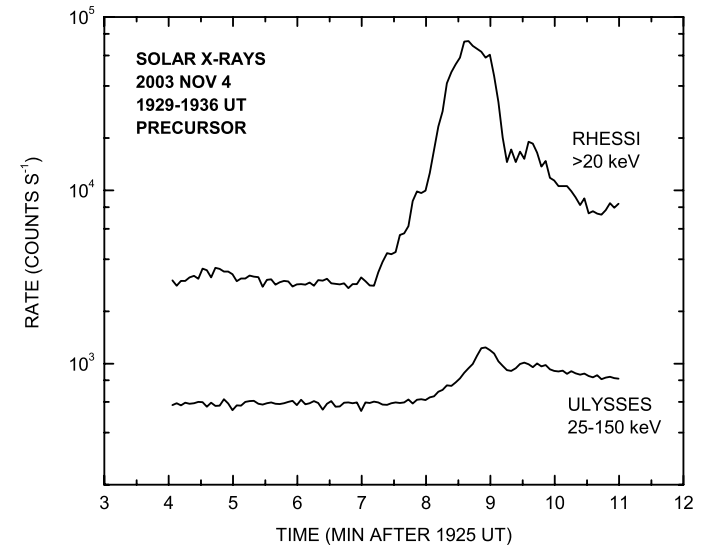

Fig. 3. The integral $(25-150 \mathrm{keV})$ counting rate of the Ulysses instrument and the counting rate of X-rays $>20 \mathrm{keV}$ observed by RHESSI during the small hard X-ray burst associated with the 2003 November 4 solar flare.

counting rates were 60.7 and 79.5 counts s ${ }^{-1}$ respectively. The observed and computed rates thus agreed within $\sim 30 \%$. (b) For the period 1934:00-1936:00 UT, which covers the decay of the first maximum, the spectral parameters were $\gamma_{1}=7.55$, $\gamma_{2}=5.61$, and $E_{\mathrm{br}}=37.3 \mathrm{keV}$. The computed and observed counting rates were 119.5 and 320.6 counts $\mathrm{s}^{-1}$ respectively. The observed rate was therefore larger than the computed rate by a factor of $\sim 2.7$.

The difference between the observed and computed counting rates of the Ulysses instrument during the decay of the small maximum represents the difference between the hard X-ray fluxes observed by Ulysses and RHESSI at that time. The difference could be caused by one or both of the following two factors: (1) directivity (anisotropy) of the hard X-ray source, and (2) partial occultation of the hard X-ray source by the photosphere from the view of the RHESSI instrument. Extensive stereoscopic observations of the directivity of flareassociated hard X-ray sources during the last solar activity cycle have shown that it is small (Kane et al. 1980, 1988, 1998). The good agreement between the observed and expected counting rates during the rise and small maximum confirm this earlier finding. Partial occultation of the hard X-ray source is therefore most likely to be the main cause of the observed difference. It appears that during the rise and first maximum, most of the hard X-ray source was located on the visible solar disk in full view of both the instruments. However, during the decay of the first maximum, there were additional components of the hard X-ray source behind the solar limb which were occulted from the view of RHESSI.

Images of the $20-30 \mathrm{keV}$ and $30-50 \mathrm{keV} \mathrm{X}$-ray sources obtained by RHESSI during the period of the maximum (1932:40-1933:40UT) and early decay (1934:00-1936:00 UT) of the small X-ray flare and late in the decay (2056:00-2102:00 UT) of the large X-ray flare are shown in Fig. 4. The solar limb is also shown. During the early rise of the small X-ray flare, the hard X-ray sources were on the disk, just inside the west limb of the Sun. On the other hand, during the early decay of that flare, the X-ray sources were just above the limb with only faint emission from the disk. During the late decay of the large $\mathrm{X}$-ray flare, the hard X-ray source was high in the corona with no detectable emission from the solar disk. It is possible, however, that the hard X-ray source extended beyond the west limb and hence a part of the source was occulted from the view of RHESSI.

During the late decay in the flare there is no detectable hard X-ray source on the solar disk. Instead, a relatively compact source is present in the corona at a height of $\sim 20000 \mathrm{~km}$ above the solar limb. This source is similar to the coronal hard X-ray sources observed by RHESSI in other solar flares (Kane \& Hurford 2003; Kane et al. 2003).

\section{Energetic electrons at the Sun}

Impulsive hard X-ray emission in solar flares is generally interpreted as thick-target bremsstrahlung produced in the chromosphere by non-thermal electrons accelerated during the flare. Several models have been developed to describe the hard X-ray source in a flare (Holman et al. 2003; McTiernan \& Petrosian 1990; Brown et al. 1971; Holman 2003). The incident hard X-ray spectrum is often a single or double power law $E^{-\gamma}$ photons $\mathrm{cm}^{-2} \mathrm{~s}^{-1} \mathrm{keV}^{-1}$, where $\mathrm{E}$ is the photon energy and the index $\gamma=2.0-6.0$, the most common value being 3.5 (Bromund et al. 1995). Ulysses observations of the integral hard X-ray counting rate can be used to estimate the energy carried by the energetic electrons at the Sun.

Since the hard X-ray spectrum incident on Ulysses is not available, we obtain constraints by first assuming the differential spectrum of the $\geqq 20 \mathrm{keV}$ photons at the time of burst maximum to be a power law with index $\gamma=3.5$. The maximum counting rate observed by Ulysses then requires the following photon spectrum at $1 \mathrm{AU}$ :

$J(E)=4.7 \times 10^{9} E^{-3.5}$ photons $\mathrm{cm}^{-2} \mathrm{~s}^{-1} \mathrm{keV}^{-1}$ at $1 \mathrm{AU}$.

For a simple thick-target model with isotropic $\mathrm{X}$-ray emission, the implied spectrum of $\geqq 20 \mathrm{keV}$ electrons at the Sun is then given by (Brown 1971; Hudson et al. 1978):

$N\left(E_{\mathrm{e}}\right)=1.8 \times 10^{44} E_{\mathrm{e}}^{-4.5}$ electrons $\mathrm{s}^{-1} \mathrm{keV}^{-1}$ at the Sun,

where $E_{\mathrm{e}}(\mathrm{keV})$ is the energy of the electrons. For electrons with energy $E_{\mathrm{e}} \geqq 20 \mathrm{keV}$, this leads to an injection rate of $1.4 \times$ $10^{39}$ electrons s $^{-1}$ into the hard X-ray source, the corresponding energy dissipation rate being $6.4 \times 10^{31} \mathrm{erg} \mathrm{s}^{-1}$. For a $\sim 200 \mathrm{~s}$ full width at half maximum for the hard X-ray time profile, we find the total number of $\geqq 20 \mathrm{keV}$ electrons accelerated during the flare to be $\sim 2.8 \times 10^{41}$. The total energy dissipated by these electrons during the flare is found to be $1.3 \times 10^{34} \mathrm{erg}$.

The above numbers represent the best estimates for this flare. Their uncertainties can be estimated by taking $\gamma$ to be 2.5 and 4.5; about $70 \%$ of the flares studied by Bromund et al. (1995) had spectral indices in this range, making it roughly equivalent to a $1 \sigma$ confidence interval. If the index $\gamma$ is assumed to be 2.5 , the total dissipated energy is found to be $4.1 \times 10^{33} \mathrm{erg}$. An assumed index of 4.5 gives $2.8 \times 10^{34} \mathrm{erg}$ for the total dissipated energy. Thus an uncertainty of \pm 1 in the 

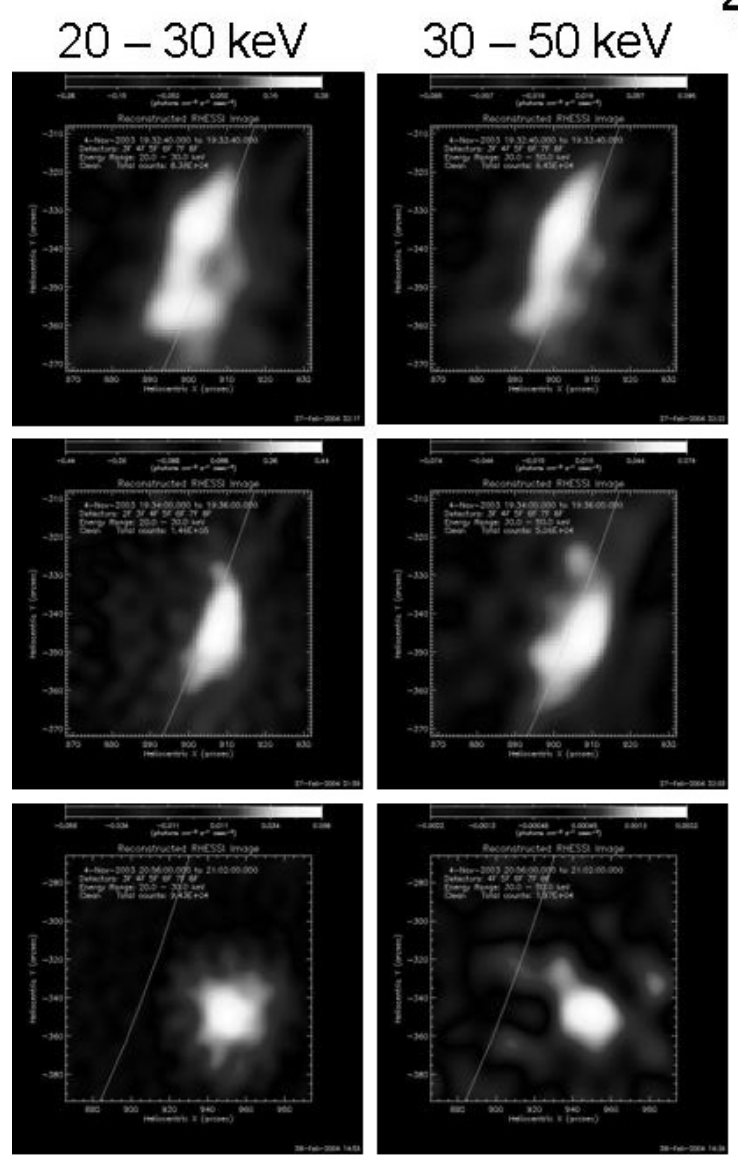

4 NOV 2003 FLARE (RHESSI)

\author{
SMALL FLARE \\ EARLY RISE \\ 1932:40-1933:40 UT
}

\author{
SMALL FLARE \\ EARLY DECAY \\ 1934:00-1936:00 UT
}

$128 \times 128$ arc-sec

Fig. 4. Images of the 20-30 keV and 30-50 keV X-ray sources obtained by RHESSI during the period of the maximum (1932:40-1933:40 UT) and early decay (1934:00-1936:00 UT) of the small X-ray flare and late in the decay (2056:00-2102:00 UT) of the large X-ray flare. The solar limb is also shown. During the early rise of the small X-ray flare, the hard X-ray sources were on the disk, just inside the west limb of the Sun. On the other hand, during the early decay of that flare, the X-ray sources were just above the limb with only faint emission from the disk. However, during the late decay of the large X-ray flare, the hard X-ray source was high in the corona with no detectable emission from the solar disk

photon spectral index corresponds to an uncertainty of a factor $\leqq 3$ in the estimates of the rate of energy dissipation and the total energy dissipated during the flare.

As stated in Sect. 4, in the case of the small burst (1933:40 UT), the photon spectrum was measured by the RHESSI instrument. The injection rate of $>20 \mathrm{keV}$ electrons into the hard X-ray source is found to be $\sim 10^{36}$ electrons s ${ }^{-1}$, the corresponding total energy dissipated by these electrons being $\sim 10^{31} \mathrm{erg}$. The production of energetic electrons in the large burst was thus $\sim 1000$ times larger than that in the small burst.

\section{Energy release and the role of the active region}

Trottet et al. (2003) have reported hard X-ray stereoscopic observations of the 1991 June 30 flare obtained by the Solar X-ray/Cosmic Gamma-Ray Burst Experiment on Ulysses and by the Burst and Transient Source Experiment on the Compton Gamma-Ray Observatory in the same energy range as the one in the present study. This is the only work to date which has allowed us to obtain information, albeit incomplete, on the spatial distribution of the hard X-ray emission and its evolution with time during a giant flare. The main conclusion of this work is that energy release during major solar flares is a complex and dynamic process which involves large spatial scales.

The flare on 2003 November 4, which produced the largest soft X-ray burst observed so far, also produced the most intense hard X-ray burst observed during the present solar activity cycle. Intense microwave emission indicates the presence of a large number of non-thermal electrons and the very high frequencies $(405 \mathrm{GHz})$ at which the radio emission could be observed indicates a very high rate of energy release during this flare. The Ulysses observations of the hard X-ray emission show that a total of $\sim 3 \times 10^{41}$ electrons with energy $\geqq 20 \mathrm{keV}$ were accelerated during this flare. The total energy carried by these electrons was between $\sim 4 \times 10^{33}$ and $3 \times 10^{34} \mathrm{erg}$. If we include the energy in the form of lower energy electrons and energetic ions the total energy released will be even larger. Also it is possible that additional energy is required to produce the flare-associated coronal mass ejection and other mass motions. This flare therefore belongs to the class of "giant" flares observed during the last solar activity cycle. 
Most models of solar flares assume that the magnetic field in the associated active region is the source of the energy released during the flare. For the active region NOAA 10486 the available energy prior to the 2003 October 29 flare has been estimated to be $\sim 8 \times 10^{33} \mathrm{erg}$ (Metcalf et al. 2004). If a comparable amount of energy was also available prior to the 2003 November 4 flare, the present observations indicate that essentially all the available energy was released during the short duration of the flare.

A study of the release and dissipation of energy during giant solar flares carried out by us earlier (Kane et al. 1995) indicated the inadequacy of the relevant active region to provide the rapid energy release necessary for the flare. Even when the total available energy in the active region is comparable to the energy released during the flare, release of all that energy during the short duration of the flare is expected to affect substantially the magnetic field structure of the active region. We are not aware of any observations which indicate large scale changes in an active region after a large flare. It is therefore possible that the instability that triggers the energy release during a solar flare affects the corona globally (rather than locally) so that resources from a substantial part of the corona inside as well as outside the active region are available for energy release and acceleration of particles.

Eleven giant solar flares were observed during the last solar activity cycle. Although only one giant flare has been observed during the present solar activity cycle, it produced one of the most intense hard X-ray bursts and the largest soft X-ray burst ever observed. The present observations therefore confirm the previous results and extend them to the present solar cycle. It therefore appears that giant flares, although rare, may be a feature of each solar cycle. However, in most of these twelve giant flares the intense X-ray flux partially or fully saturated the soft $\mathrm{X}$-ray and hard X-ray instruments located at $\sim 1 \mathrm{AU}$ from the Sun. The observations presented here have demonstrated very clearly the need for large dynamic ranges for the soft X-ray and hard X-ray instruments intended for solar flare observations. It is hoped that this need will receive full consideration in the design of the future X-ray instrumentation so that reliable and accurate observations of the most energetic, giant, solar flares are possible.
Acknowledgements. The authors are thankful to Dr. G. Trottet for his comments and suggestions, to R. Williams and J. Selvidge for their help in processing the Ulysses data, and to the RHESSI team for providing excellent data and data analysis tools. S. R. Kane and J. M. McTiernan were supported by NASA contract NAS 5-98033. K. Hurley and the Ulysses data analysis were supported by NASA under JPL contract 958056.

\section{References}

Barat, C., Trottet, G., Vilmer, N., et al. 1994, ApJ, 425, L109

Bromund K., McTiernan, J. M., \& Kane, S. R. 1995, ApJ, 455, 733

Brown, J. C. 1971, Sol. Phys., 18, 489

Brown, J. C., Emslie, A. G., \& Kontar, E. P. 2003, ApJ, 595, L115

Holman, G. D. 2003, ApJ, 586, 606

Holman, G. D., Kundu, M. R., \& Kane, S. R. 1989, ApJ, 345, 1050

Hudson, H. S., Canfield, R. C., \& Kane, S. R. 1978, Sol. Phys., 60, 137

Hurley, K., Sommer, M., Atteia, J.-L., et al. 1992, A\&AS, 92, 401

Kahler, S., 1992, ARA\&A, 30, 113

Kane, S. R., \& Hurford, G. J. 2003, Adv. Space Res., 32, 2489

Kane, S. R., Anderson, K. A., Evans, W. D., Klebesadel, R. W., \& Laros, J. G. 1980, ApJ, 239, L85

Kane, S. R., Fenimore, E. E., Klebesadel, R. W., \& Laros, J. G. 1988, ApJ, 326, 1017

Kane, S. R., Hurley, K, McTiernan, J. M., \& Sommer, M. 1993, Adv. Space Res., 13, 241

Kane, S. R., Hurley, K., McTiernan, J. M., et al. 1995, ApJ, 446, L47

Kane, S. R., Hurley, K., McTiernan, J. M., et al., 1998, ApJ, 500, 1003

Kane, S. R., Sawant, H. S., Cecatto, J. R., et al. 2003, Adv. Space Res., 32,2503

Kaufmann, P., Raulin, J., de Castro, C. G. G., et al., 2004, ApJ, 603, L121

Kiplinger, A. L., \& Garcia, H. A. 2004, BAAS, 36, 739

Lin, R. P., \& Hudson, H. S. 1976, Sol. Phys., 50, 153

Lin, R. P., Dennis, B. R., \& Benz, A. O. 2002, Sol. Phys., 210, 1

McTiernan, J. M., \& Petrosian, V. 1990, ApJ, 359, 541

Metcalf, T., Leka, K. D., Mickey, D., LaBonte, B., \& Ryder, L. 2004, Presentation at Univ of California, Berkeley and BAAS, 36, 668

Trottet, G., Schwartz, R., Hurley, K., et al. 2003, A\&A, 403, 1157

Vilmer, N., Trottet, G., Barat, C. et al. 1999, A\&A, 342, 575 